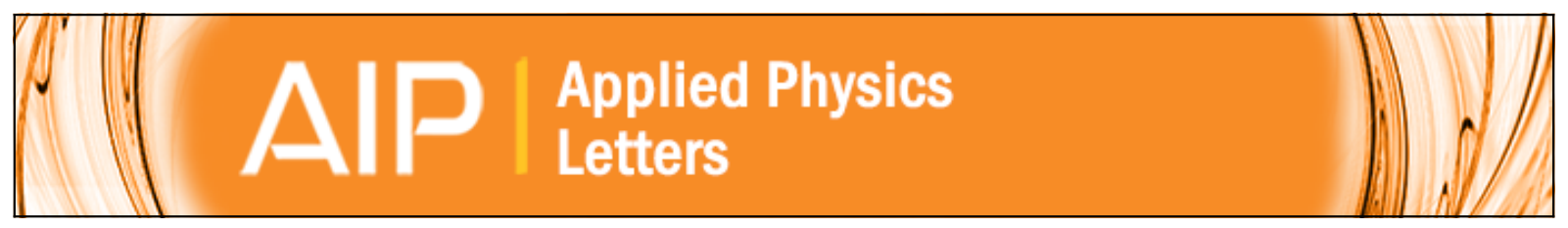

\title{
Large g-factor enhancement in high-mobility InAs/AISb quantum wells
}

Yu. G. Sadofyev, A. Ramamoorthy, B. Naser, J. P. Bird, S. R. Johnson, and Y.-H. Zhang

Citation: Applied Physics Letters 81, 1833 (2002); doi: 10.1063/1.1504882

View online: http://dx.doi.org/10.1063/1.1504882

View Table of Contents: http://scitation.aip.org/content/aip/journal/apl/81/10?ver=pdfcov

Published by the AIP Publishing

\section{Articles you may be interested in}

The effective g-factor in In0.53Ga0.47As/In0.52Al0.48As quantum well investigated by magnetotransport measurement

J. Appl. Phys. 113, 033704 (2013); 10.1063/1.4776236

Magnetotransport properties of two-dimensional electron gas in AlSb/InAs quantum well structures designed for device applications

J. Appl. Phys. 96, 6353 (2004); 10.1063/1.1792385

Asymmetric AlAsSb/lnAs/CdMgSe quantum wells grown by molecular-beam epitaxy

Appl. Phys. Lett. 84, 4777 (2004); 10.1063/1.1759777

Mobility enhancement by reduced remote impurity scattering in a pseudomorphic In $0.7 \mathrm{Ga} 0.3 \mathrm{As} / \mathrm{In} 0.52 \mathrm{Al} 0.48$ As quantum well high electron mobility transistor structure with (411)A super-flat interfaces grown by molecularbeam epitaxy

J. Vac. Sci. Technol. B 19, 1515 (2001); 10.1116/1.1387454

Transport and quantum electron mobility in the modulation $\mathrm{Si}$-doped pseudomorphic GaAs/ln $0.2 \mathrm{Ga} 0.8 \mathrm{As} / \mathrm{Al}$ $0.2 \mathrm{Ga} 0.8$ As quantum well grown by metalorganic vapor phase epitaxy

Appl. Phys. Lett. 77, 999 (2000); 10.1063/1.1289035 


\title{
Large $\boldsymbol{g}$-factor enhancement in high-mobility InAs/AISb quantum wells
}

\author{
Yu. G. Sadofyev, A. Ramamoorthy, B. Naser, J. P. Bird, ${ }^{\text {a) }}$ S. R. Johnson, and Y.-H. Zhang \\ Department of Electrical Engineering and Center for Solid State Electronic Research, Arizona State \\ University, Tempe, Arizona 85287-5706
}

(Received 13 June 2002; accepted for publication 10 July 2002)

\begin{abstract}
We discuss the growth by molecular-beam epitaxy, and studies of the low-temperature electrical properties, of undoped InAs/AlSb quantum wells. The two-dimensional electron gas realized in the wells exhibits high mobility at low temperatures, and an analysis of its Shubnikov-de Haas oscillations suggests this mobility is limited by scattering from remotely located unintentional dopants. Spin splitting of the oscillations is clearly resolved at $4.2 \mathrm{~K}$, revealing a $g$-factor as large as -60 at high magnetic fields. The size of this enhancement increases with decreasing electron density, and is thought to reflect the associated increase in the strength of the effective Coulomb interaction. (C) 2002 American Institute of Physics. [DOI: 10.1063/1.1504882]
\end{abstract}

The InAs/AlSb quantum-well (QW) system is of great interest for application in high-speed transistors, since the small effective mass for electrons in the InAs well gives rise to high carrier mobility and velocity, and to strong quantization of the electronic energy spectrum. ${ }^{1-12}$ The large conduction-band offset between InAs and AlSb also results in the formation of a high barrier at the interface between these materials, and so allows for strong confinement of electrons in such QWs. With its much larger $g$-factor $(-15)$ than either Si or GaAs, high-mobility InAs is furthermore of interest in the emerging area of spintronics, where the objective is to develop device structures that exploit the spin degree of freedom of the charge carriers to realize novel functionality.

In this letter, we report on the growth of high-mobility InAs QWs by molecular-beam epitaxy (MBE), and discuss the results of studies of their low-temperature transport properties. The two-dimensional electron gas (2DEG) realized in the undoped wells exhibits high mobility at low temperatures $\left(\leqslant 4.6 \times 10^{5} \mathrm{~cm}^{2} / \mathrm{Vs}\right.$ at $\left.4.2 \mathrm{~K}\right)$, and a study of the Shubnikov-de Haas ( $\mathrm{SdH}$ ) oscillations in its magnetoresistance suggests this mobility is limited by scattering from remotely located ionized dopants. Spin splitting of the oscillations is clearly resolved at $4.2 \mathrm{~K}$, revealing evidence for a strong enhancement of the $g$-factor. Our analysis ${ }^{13}$ suggests that the value of this parameter can be as large as -60 at high magnetic fields, and that the size of this enhancement decreases with increasing electron density. This scaling is reminiscent of that found in $\mathrm{Si}$ inversion layers, ${ }^{14}$ and is thought to reflect the increase in the strength of the effective Coulomb interaction with decreasing density.

Undoped InAs QWs were grown by MBE on (100) semi-insulating GaAs and $p$-GaSb substrates. For growth on the GaSb substrates, a $2.4 \mu \mathrm{m} \mathrm{GaSb}$ buffer was grown directly on the wafer (samples A678 and A679). For growth on the GaAs substrates, and in order to accommodate the much larger lattice constant of InAs, metamorphic AlSb (or GaSb) layers were deposited on a $10 \mathrm{~nm}$ AlAs layer, ${ }^{6}$ grown on a $200 \mathrm{~nm} \mathrm{GaAs}$ buffer. The metamorphic growth was initiated

${ }^{a)}$ Electronic mail: bird@asu.edu with the deposition of $100 \mathrm{~nm}$ of $\mathrm{AlSb}$ at $570^{\circ} \mathrm{C}$, followed by a $2.4 \mu \mathrm{m}$ metamorphic AlSb buffer layer grown at $570{ }^{\circ} \mathrm{C}$ (samples A839 and A856), or a GaSb buffer layer grown at $510^{\circ} \mathrm{C}$ (sample B824). The smaller lattice constant of the GaSb buffer (compared to an AlSb buffer) reduces the tensile strain of the InAs QW, while the growth on a GaSb substrate (as opposed to a GaAs substrate) virtually eliminates the threading-dislocation density in the InAs QW. ${ }^{5}$ Although the $\mathrm{GaSb}$ is conducting at room temperature, its carriers freeze out at liquid helium temperatures, ensuring that conduction is limited to the InAs QW. For all samples, the GaSb (or AlSb) buffer was followed by a ten-period GaSb $(2.5 \mathrm{~nm}) / \mathrm{AlSb}$ $(2.5 \mathrm{~nm})$ superlattice grown at $480-490^{\circ} \mathrm{C}$, a $12 \mathrm{~nm} \mathrm{AlSb}$ barrier, a $15 \mathrm{~nm}$ InAs QW, a 30-40 nm AlSb, AlGaSb, or AlSbAs barrier, and a $6 \mathrm{~nm} \mathrm{GaSb}$ cap. The shutter sequences employed at the start and the finish of the InAs QW enabled the formation of InSb-like bonds at both interfaces of the InAs layer. ${ }^{1}$ The substrate-heater temperature was decreased by $20^{\circ} \mathrm{C}$ during the InAs QW growth, in order to achieve a constant substrate temperature $\left(480^{\circ} \mathrm{C}\right)$. The growth rates were $6 \mathrm{~nm} / \mathrm{min}$ for the InAs and $12 \mathrm{~nm} / \mathrm{min}$ for the Sbcontaining layers. The $\mathrm{Sb}: \mathrm{Ga}(\mathrm{Al})$ flux ratio was $1.2: 1$ for all Sb-containing layers, while the $\mathrm{As}_{2}$ : In flux ratio was 2.0:1. The different top barriers allow for the formation of symmetric $(\mathrm{AlSb})$ and asymmetric $\left(\mathrm{Al}_{0.8} \mathrm{Ga}_{0.2} \mathrm{Sb}\right)$ wells, while the $\left(\mathrm{AlSb} / \mathrm{AlSb}_{0.9} \mathrm{As}_{0.1}\right)$ barrier is expected to yield a reduced hole-leakage current in field-effect structures based on the InAs QW. All layers exhibited mirrorlike surfaces and good surface reconstructions; $(1 \times 3)$ for the $(\mathrm{Al}, \mathrm{Ga}) \mathrm{Sb}$, and weak $(2 \times 4)$ for the InAs. The parameters of the different samples are listed in Table I.

After growth, the wafers were diced into rectangular bars with approximate dimensions of $15 \times 4 \mathrm{~mm}^{2}$, and ohmic contact to the 2DEG layers was made by soldering indium contacts in a Hall-bar arrangement. The samples were then wire bonded into chip carriers, and standard lock-in techniques were used to measure their magnetoresistance at 4.2 $\mathrm{K}$ in an immersion-bath cryostat. One of the samples (A839) was also investigated at temperatures between 0.01 and $8 \mathrm{~K}$, in a dilution refrigerator. In order to avoid unwanted heating effects, the drive current used in all measurements was kept 
TABLE I. Upper-barrier composition and transport properties of the different samples.

\begin{tabular}{|c|c|c|c|c|c|c|c|c|}
\hline & $\begin{array}{l}\text { Top } \\
\text { Barrier }\end{array}$ & $\begin{array}{c}\mu_{300 \mathrm{~K}} \\
\left(\mathrm{~cm}^{2} / \mathrm{Vs}\right)\end{array}$ & $\begin{array}{c}n_{300 \mathrm{~K}} \\
\left(\times 10^{11} \mathrm{~cm}^{-2}\right)\end{array}$ & $\begin{array}{c}\mu_{4.2 \mathrm{~K}} \\
\left(\mathrm{~cm}^{2} / \mathrm{Vs}\right)\end{array}$ & $\begin{array}{c}n_{4.2 \mathrm{~K}, \text { Hall }} \\
\left(\times 10^{11} \mathrm{~cm}^{-2}\right)\end{array}$ & $\begin{array}{c}n_{4.2 \mathrm{~K}}, \mathrm{SdH} \\
\left(\times 10^{11} \mathrm{~cm}^{-2}\right)\end{array}$ & $\begin{array}{c}\tau_{q} \\
(\mathrm{ps})\end{array}$ & $\begin{array}{c}\tau / \tau_{q} \\
@ 4.2 \mathrm{~K}\end{array}$ \\
\hline A839 & $\mathrm{AlSb}, 40 \mathrm{~nm}$ & 30540 & 11.5 & 184550 & 5.53 & 5.12 & 0.10 & 25 \\
\hline A856 & $\begin{array}{l}\mathrm{Al}_{0.8} \mathrm{Ga}_{0.2} \mathrm{Sb} \\
\quad 40 \mathrm{~nm}\end{array}$ & 27420 & 11.7 & 444350 & 5.01 & 5.27 & 0.11 & 55 \\
\hline B824 & $\mathrm{AlSb}, 30 \mathrm{~nm}$ & 28510 & 15.7 & 413600 & 7.45 & 6.56 & 0.11 & 51 \\
\hline A678 & $\begin{array}{c}\text { AlSb, } 15 \mathrm{~nm}+ \\
\mathrm{AlSb}_{0.9} \mathrm{As}_{0.1} \\
15 \mathrm{~nm}\end{array}$ & - & - & 460400 & 8.09 & 6.97 & 0.16 & 39 \\
\hline A679 & AlSb, $30 \mathrm{~nm}$ & - & - & 269850 & 8.62 & 6.60 & 0.11 & 33 \\
\hline
\end{tabular}

to just a few nA. The samples were cooled in the dark with their contacts grounded, and the measurements reported here were performed without any low-temperature illumination.

In Table I, we summarize the results of measurements of the electrical properties of the QW samples. The table shows that mobilities as high as $4.6 \times 10^{5} \mathrm{~cm}^{2} / \mathrm{Vs}$ are achieved at low temperatures, where typical carrier densities $\left(n_{s}\right)$ in the undoped samples are in the range of $5-7 \times 10^{11} \mathrm{~cm}^{-2}$. Assuming the usual effective mass for InAs $\left(m^{*}=0.024 m_{0}\right.$, where $m_{0}$ is the free-electron mass), the range of lowtemperature mobilities listed in Table I implies a variation of the relaxation time $(\tau)$ from 2.5 to 6.3 ps. In Fig. 1, we show the measured magnetoresistance of four QW samples, and see that spin splitting of the $\mathrm{SdH}$ oscillations is apparent at magnetic fields as low as a couple of Tesla. The periodicity of these oscillations yields an estimate for $n_{s}$ that we find to be about $5 \%$ smaller than that inferred from the result of Hall measurements (Table I), indicating the presence of an additional, low-density, channel for conduction. A similar discrepancy was noted in the recent study by Brosig et al., ${ }^{11}$ although these authors did not comment on its origin. The difference between the Hall and $\mathrm{SdH}$ measurements increases with increasing $2 \mathrm{DEG}$ density, leading us to suggest it may result from weak filling of the second subband in the QW. Since beating of the SdH oscillations is not observed in Fig. 1 (with the possible exception of the curve for sample

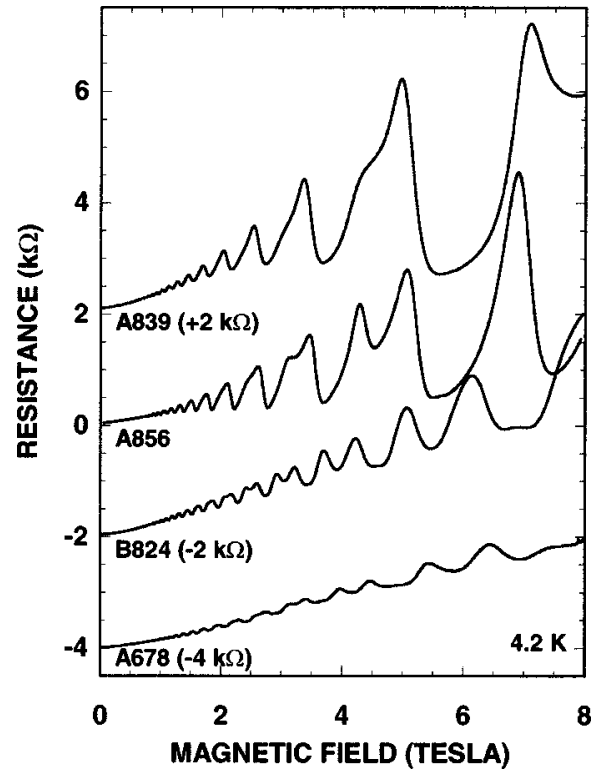

FIG. 1. Magnetoresistance of four different QWs (indicated) at $4.2 \mathrm{~K}$.
A839), the mobility in this second subband appears to be very low.

By analyzing the line shape of the $\mathrm{SdH}$ oscillations at low magnetic fields, ${ }^{15}$ it was possible to determine the value of the quantum lifetime $\left(\tau_{q}\right)$, which essentially corresponds to the total electron scattering rate. Once again assuming the bulk effective mass for InAs, the value of $\tau_{q}$ is found to vary from 0.1 to $0.2 \mathrm{ps}$ in the different samples (Table I), yielding a corresponding ratio $\tau / \tau_{q} \sim 25-55$ (Table I). Such large values for this ratio are usually associated with scattering from the long-range potential established by remotely located ionized dopants, and are consistent with current opinion on these structures, which suggests that their carriers originate in the barriers, or are provided by surface or interface states. $^{3,5}$ In Fig. 2, we show the influence of temperature on the magnetoresistance of one of the samples, and see that the $\mathrm{SdH}$ oscillations become increasingly resolved with decreasing temperature. In spite of this, however, our analysis indicates that the values of $\tau$ and $\tau_{q}$ do not change significantly over the same temperature range. This observation is consistent with our earlier assertion that the mobility is limited by scattering from remotely located ionized dopants. ${ }^{16}$

With the $\mathrm{SdH}$ oscillations resolved, it is possible to obtain an estimate for the effective $g$-factor from the magnitude

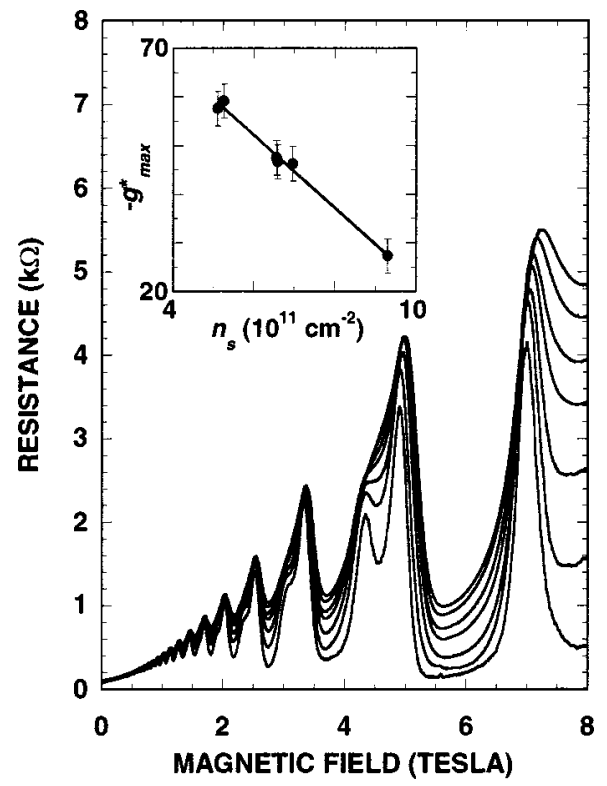

FIG. 2. Main panel: Temperature dependence of the $\mathrm{SdH}$ oscillations in sample A839. From top to bottom, temperatures are: 7.3, 6.3, 4.2, 3.3, 1.7, 0.58 , and $0.02 \mathrm{~K}$ Inset: variation of the maximum $g$-factor vith carrier density $\left(n_{s}\right)$ in the different QWs. Solid line is a guide to the eye. 


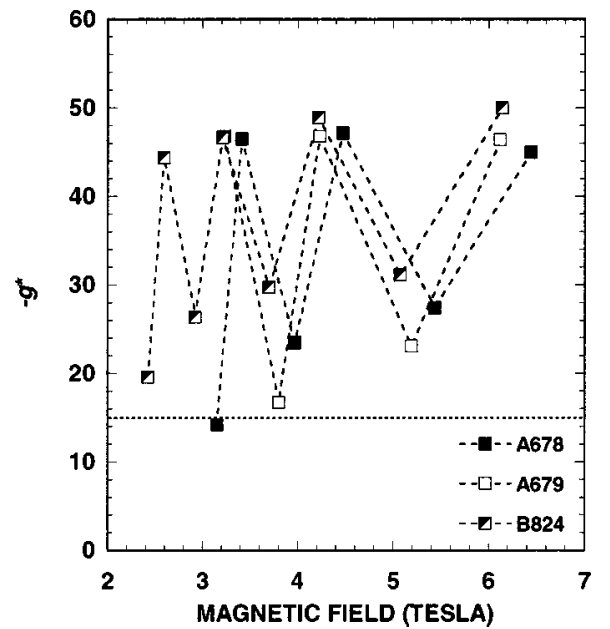

FIG. 3. Variation of the $g$-factor with magnetic field, inferred for three different QWs (indicated), using the method of Ref. 12. The dotted line indicates the value of the $g$-factor at zero magnetic field in bulk InAs.

of their spin splitting. The approach we employ here is discussed in detail in Ref. 13, where it was noted that the magnetic fields $\left(B_{N}^{ \pm}\right)$at which the spin-resolved maxima in the $\mathrm{SdH}$ oscillations occur are defined by

$$
\frac{\hbar^{2} \pi n_{s}}{m^{*}}=\left[N_{L}+\frac{1}{2}\right] \frac{\hbar e}{m^{*}} B_{N}^{ \pm} \pm \frac{1}{2} g^{*} \mu_{B} B_{N}^{ \pm},
$$

where $N_{L}$ is the Landau-level index, $g^{*}$ is the effective $g$-factor, and $\mu_{B}$ is the Bohr magneton. In Fig. 3, we plot the variation of the $g$-factor [determined from Eq. (1)] with magnetic field for three different QWs. The oscillations in this figure are associated with the depopulation of successive, spin-resolved, Landau levels, which causes a modulation of the strength of the exchange interaction known to give rise to the $g$-factor enhancement. ${ }^{17}$ This interaction is weakest when the Fermi level lies between two different Landau levels, but is maximal when it lies between the spin-resolved subbands of any Landau level. ${ }^{17}$ The oscillations of the $g$-factor shown in Fig. 3 are indeed consistent with these arguments, and we furthermore note that the oscillation minima correspond, roughly, to the bulk $g$-factor in InAs (dotted line). It is clear from Fig. 3 that there is a sample-dependent variation of the $g$-factor enhancement, and in the inset to Fig. 2 we plot the variation of the maximum $g$-factor with the electron density. (The data point at $9.3 \times 10^{11} \mathrm{~cm}^{-2}$ was obtained in measurements of an InAs/AlGaSb heterojunction, featuring a $15 \mathrm{~nm}$ InAs QW. ${ }^{18}$ ) The $g$-factor enhancement clearly decreases with increasing carrier density, and a similar effect has been found in studies of $\mathrm{Si}$ inversion layers, ${ }^{13}$ where it has been argued to result from a decrease in the strength of the effective Coulomb interaction with decreasing density. ${ }^{19}$ To the best of our knowledge, however, this effect has not been studied previously in the InAs/AlSb QW system.

In conclusion, we have discussed the MBE growth, and low-temperature electrical characterization, of undoped InAs/AlSb QWs. The 2DEG realized in the wells exhibits high mobility at low temperatures, and an analysis of its $\mathrm{SdH}$ oscillations suggests this mobility is limited by scattering from remotely located unintentional dopants. Spin splitting of the oscillations is clearly resolved at $4.2 \mathrm{~K}$, revealing a $g$-factor as large as -60 at high magnetic fields. The size of this enhancement increases with decreasing electron density, and is thought to reflect the associated increase in the strength of the effective Coulomb interaction.

This work is supported by the Office of Naval Research, the Department of Energy NSET program, and Arizona State University.

${ }^{1}$ G. Tuttle, H. Kroemer, and J. H. English, J. Appl. Phys. 67, 3032 (1990).

${ }^{2}$ P. F. Hopkins, A. J. Rimberg, R. M. Westervelt, G. Tuttle, and H. Kroemer, Appl. Phys. Lett. 58, 1428 (1991).

${ }^{3}$ S. Ideshita, A. Furukawa, Y. Mochizuki, and M. Mizuta, Appl. Phys. Lett. 60, 2549 (1991).

${ }^{4}$ J. Scriba, S. Seitz, A. Wixforth, J. P. Kotthaus, G. Tuttle, J. H. English, and H. Kroemer, Surf. Sci. 267, 483 (1992).

${ }^{5}$ H. Kroemer, C. Nguyen, and B. Bar, J. Vac. Sci. Technol. 10, 1769 (1992).

${ }^{6}$ C. Nguyen, B. Brar, C. R. Bolognesi, J. J. Pekarik, H. Kroemer, and J. H. English, J. Electron. Mater. 22, 255 (1993).

${ }^{7}$ C. Tedesco, E. Zanoni, C. Canali, S. Bigliardi, M. Manfredi, D. C. Streit, and W. T. Anderson, IEEE Trans. Electron Devices 40, 1211 (1993).

${ }^{8}$ T. Maemoto, H. Dobashi, S. Izumiya, K. Yoh, and M. Inoue, Jpn. J. Appl. Phys. 33, 7204 (1994).

${ }^{9}$ J. B. Boos, W. Kruppa, B. R. Bennett, D. Park, S. W. Kirchoefer, R. Bass, and H. B. Dietrich, IEEE Trans. Electron Devices 45, 1869 (1998).

${ }^{10}$ B. R. Bennett, M. J. Yang, B. V. Shanabrook, J. B. Boos, and D. Park, Appl. Phys. Lett. 72, 1193 (1998).

${ }^{11}$ S. Brosig, K. Ensslin, B. Brar, M. Thomas, and H. Kroemer, Physica E (Amsterdam) 2, 214 (1998).

${ }^{12}$ M. J. Yang, K. A. Cheng, C. H. Yang, and J. C. Culbertson, Appl. Phys. Lett. 80, 1201 (2002).

${ }^{13}$ A. Raymond, J. L. Robert, C. Bousquet, W. Zawadzki, F. Alexandre, and I. M. Masson, Solid State Commun. 55, 271 (1985).

${ }^{14}$ F. F. Fang and P. J. Stiles, Phys. Rev. 174, 823 (1968).

${ }^{15}$ T. Ando, A. B. Fowler, and F. Stern, Rev. Mod. Phys. 54, 437 (1982).

${ }^{16}$ J. H. English, A. C. Gossard, H. L. Stormer, and K. W. Baldwin, Appl. Phys. Lett. 50, 1826 (1987).

${ }^{17}$ T. Ando, in High Magnetic Fields in Semiconductor Physics II, edited by G. Landwehr (Springer, Berlin, 1989) pp. 164-173.

${ }^{18}$ T. Maemoto, T. Kobayashi, T. Karasaki, K. Kita, S. Sasa, M. Inoue, K. Ishibashi, and Y. Aoyagi, Physica B 314, 481 (2002).

${ }^{19}$ J. F. Janak, Phys. Rev. 178, 1416 (1969). 